\title{
Correction: Fluorescent core-shell nanoparticles and nanocapsules using comb-like macromolecular RAFT agents: synthesis and functionalization thereof
}

Cite this: Polym. Chem., 2016, 7, 4284

\author{
Chloé Grazon, ${ }^{* a}$ Jutta Rieger, ${ }^{* b}$ Patricia Beaunier, ${ }^{c}$ Rachel Méallet-Renault ${ }^{\mathrm{a}}$ and \\ Gilles Clavier ${ }^{a}$
}

DOI: $10.1039 / c 6 p y 90091 \mathrm{~g}$

Correction for 'Fluorescent core-shell nanoparticles and nanocapsules using comb-like macromolecular RAFT agents: synthesis and functionalization thereof' by Chloé Grazon, et al., Polym. Chem., 2016, DOI: 10.1039/c6py00646a.

www.rsc.org/polymers

The authors regret the error in the brightness values in Table 2 in the original manuscript. The corrected version of Table 2 is as shown below.

Table 2 Physico-chemical properties of FNP with different shells, recorded in water at RT

\begin{tabular}{|c|c|c|c|c|c|c|c|c|c|c|c|c|}
\hline Shell & FNP & $n_{\pi}{ }^{a}$ & $\begin{array}{l}{[\pi]^{b},} \\
\text { mol L }_{\mathrm{S}}{ }^{-1}\end{array}$ & $N_{\pi}^{c}$ & \multicolumn{4}{|c|}{ Fluorescence properties } & $\begin{array}{l}\text { DLS } \\
\begin{array}{l}D_{z}(\sigma)^{g} \\
\mathrm{~nm}\end{array}\end{array}$ & \multicolumn{3}{|l|}{ TEM } \\
\hline $\mathrm{PPEOA}_{11}$ & FNP2 & 1.3 & 0.08 & - & 0.58 & 4.9 & 970 & - & $110(0.18)$ & - & - & - \\
\hline $\mathrm{P}\left(\mathrm{AA}_{6}-\mathrm{Co}-\mathrm{PEOA}_{6}\right)$ & FNP3 & 1.8 & 0.13 & - & 0.44 & 4.2 & 1230 & - & $85(0.19)$ & - & - & - \\
\hline $\mathrm{P}\left(\mathrm{AA}_{11}-c o-\mathrm{PEOA}_{11}\right)$ & FNP5 & 1.9 & 0.10 & 1960 & 0.52 & 4.5 & 1300 & 7.4 & $85(0.10)$ & 37 & 1030 & 0.24 \\
\hline $\mathrm{PEO}_{45}-b-\mathrm{PAA}_{15}{ }^{k}$ & FNP10 & 2.1 & 0.17 & 3680 & 0.24 & 3.2 & 980 & 6.4 & $80(0.15)$ & 40 & 1750 & 0.35 \\
\hline
\end{tabular}

${ }^{a}$ Average number of BODIPY monomers $(\pi)$ per polymer chain. ${ }^{b}$ Molar concentration of BODIPY monomers $(\pi)$ per liter of styrene. ${ }^{c}$ Average number of $\pi$ per nanoparticles determined using eqn (SI-3). ${ }^{d}$ Average decay time, determined using eqn (1) $\left(\lambda_{\mathrm{exc}}=495 \mathrm{~nm}, \lambda_{\mathrm{F}}=543 \mathrm{~nm}\right)$. ${ }^{e}$ Fluorescence spectra full width at half maximum $\left(\Delta_{1 / 2}\right) \cdot{ }^{f}$ Estimated nanoparticle brightness eqn (2). ${ }^{g} z$-Average particle diameter measured by DLS. ${ }^{h}$ FNPs' core diameter estimated by TEM. ${ }^{i}$ Aggregation number calculated with eqn (SI-4) based on TEM diameters. ${ }^{j}$ Density of the polymer chains on the FNPs' surface (eqn (SI-4)). ${ }^{k}$ Already published results. ${ }^{52}$

The Royal Society of Chemistry apologises for these errors and any consequent inconvenience to authors and readers. 